\title{
Some Inequalities Involving Perimeter and Torsional Rigidity
}

\author{
Luca Briani $^{1} \cdot$ Giuseppe Buttazzo ${ }^{1}\left[\right.$ Francesca Prinari $^{2}$ \\ Accepted: 10 October 2020 / Published online: 21 October 2020 \\ (c) The Author(s) 2020
}

\begin{abstract}
We consider shape functionals of the form $F_{q}(\Omega)=P(\Omega) T^{q}(\Omega)$ on the class of open sets of prescribed Lebesgue measure. Here $q>0$ is fixed, $P(\Omega)$ denotes the perimeter of $\Omega$ and $T(\Omega)$ is the torsional rigidity of $\Omega$. The minimization and maximization of $F_{q}(\Omega)$ is considered on various classes of admissible domains $\Omega$ : in the class $\mathcal{A}_{\text {all }}$ of all domains, in the class $\mathcal{A}_{\text {convex }}$ of convex domains, and in the class $\mathcal{A}_{\text {thin }}$ of thin domains.
\end{abstract}

Keywords Torsional rigidity $\cdot$ Shape optimization $\cdot$ Perimeter $\cdot$ Convex domains

Mathematics Subject Classification 49Q10 - 49J45 - 49R05 $\cdot 35 \mathrm{P} 15 \cdot 35 \mathrm{~J} 25$

\section{Introduction}

In this paper, given an open set $\Omega \subset \mathbb{R}^{d}$ with finite Lebesgue measure, we consider the quantities

$$
\begin{aligned}
& P(\Omega)=\text { perimeter of } \Omega ; \\
& T(\Omega)=\text { torsional rigidity of } \Omega .
\end{aligned}
$$

$\varangle$ Giuseppe Buttazzo

giuseppe.buttazzo@unipi.it

http://www.dm.unipi.it/pages/buttazzo/

Luca Briani

luca.briani@phd.unipi.it

Francesca Prinari

francescaagnese.prinari@unife.it

http://docente.unife.it/francescaagnese.prinari/

1 Dipartimento di Matematica, Università di Pisa, Largo B. Pontecorvo 5, 56127 Pisa, Italy

2 Dipartimento di Matematica e Informatica, Università di Ferrara, Via Machiavelli 30, 44121 Ferrara, Italy 
The perimeter $P(\Omega)$ is defined according to the De Giorgi formula

$$
P(\Omega)=\sup \left\{\int_{\Omega} \operatorname{div} \phi d x: \phi \in C_{c}^{1}\left(\mathbb{R}^{d} ; \mathbb{R}^{d}\right),\|\phi\|_{L^{\infty}\left(\mathbb{R}^{d}\right)} \leq 1\right\} .
$$

The scaling property of the perimeter is

$$
P(t \Omega)=t^{d-1} P(\Omega) \quad \text { for every } t>0
$$

and the relation between $P(\Omega)$ and the Lebesgue measure $|\Omega|$ is the well-known isoperimetric inequality:

$$
\frac{P(\Omega)}{|\Omega|^{(d-1) / d}} \geq \frac{P(B)}{|B|^{(d-1) / d}}
$$

where $B$ is any ball in $\mathbb{R}^{d}$. In addition, the inequality above becomes an equality if and only if $\Omega$ is a ball (up to sets of Lebesgue measure zero).

The torsional rigidity $T(\Omega)$ is defined as

$$
T(\Omega)=\int_{\Omega} u d x
$$

where $u$ is the unique solution of the PDE

$$
\left\{\begin{array}{l}
-\Delta u=1 \quad \text { in } \Omega \\
u \in H_{0}^{1}(\Omega)
\end{array}\right.
$$

Equivalently, $T(\Omega)$ can be characterized through the maximization problem

$$
T(\Omega)=\max \left\{\left[\int_{\Omega} u d x\right]^{2}\left[\int_{\Omega}|\nabla u|^{2} d x\right]^{-1}: u \in H_{0}^{1}(\Omega) \backslash\{0\}\right\} .
$$

Moreover $T$ is increasing with respect to the set inclusion, that is

$$
\Omega_{1} \subset \Omega_{2} \Longrightarrow T\left(\Omega_{1}\right) \leq T\left(\Omega_{2}\right)
$$

and $T$ is additive on disjoint families of open sets. The scaling property of the torsional rigidity is

$$
T(t \Omega)=t^{d+2} T(\Omega), \quad \text { for every } t>0,
$$

and the relation between $T(\Omega)$ and the Lebesgue measure $|\Omega|$ is the well-known Saint-Venant inequality (see for instance $[16,17]$ ):

$$
\frac{T(\Omega)}{|\Omega|^{(d+2) / d}} \leq \frac{T(B)}{|B|^{(d+2) / d}}
$$


Again, the inequality above becomes an equality if and only if $\Omega$ is a ball (up to sets of capacity zero). If we denote by $B_{1}$ the unitary ball of $\mathbb{R}^{d}$ and by $\omega_{d}$ its Lebesgue measure, then the solution of (1.2), with $\Omega=B_{1}$, is

$$
u(x)=\frac{1-|x|^{2}}{2 d}
$$

which provides

$$
T\left(B_{1}\right)=\frac{\omega_{d}}{d(d+2)}
$$

We are interested in the problem of minimizing or maximizing quantities of the form

$$
P^{\alpha}(\Omega) T^{\beta}(\Omega)
$$

on some given class of open sets $\Omega \subset \mathbb{R}^{d}$ having a prescribed Lebesgue measure $|\Omega|$, where $\alpha, \beta$ are two given exponents. Similar problems have been considered for shape functionals involving:

- the torsional rigidity and the first eigenvalue of the Laplacian in $[2,3,6,8,11,19,20$, 22];

- the torsional rigidity and the Newtonian capacity in [1];

- the perimeter and the first eigenvalue of the Laplacian in [14];

- the perimeter and the Newtonian capacity in $[9,13]$.

The case $\beta=0$ reduces to the isoperimetric inequality, and we have, denoting by $\Omega_{m}^{*}$ a ball of measure $m$,

$$
\left\{\begin{array}{l}
\min \{P(\Omega):|\Omega|=m\}=P\left(\Omega_{m}^{*}\right) \\
\sup \{P(\Omega):|\Omega|=m\}=+\infty
\end{array}\right.
$$

Similarly, in the case $\alpha=0$, the Saint Venant inequality yields

$$
\max \{T(\Omega):|\Omega|=m\}=T\left(\Omega_{m}^{*}\right)=\frac{m}{d(d+2)}\left(\frac{m}{\omega_{d}}\right)^{2 / d}
$$

while

$$
\inf \{T(\Omega):|\Omega|=m\}=0
$$

Indeed if we choose $\Omega_{n}=\cup_{k=1}^{n} B_{n, k}$ where $B_{n, k}$ are disjoint balls of measure $\mathrm{m} / n$ each, we get for every $n \in \mathbb{N}$

$$
\inf \{T(\Omega):|\Omega|=m\} \leq T\left(\Omega_{n}\right)=\frac{m^{(d+2) / d}}{d(d+2) \omega_{d}^{2 / d}} n^{-2 / d} \rightarrow 0 .
$$


The case when $\alpha$ and $\beta$ have a different sign is also immediate; for instance, if $\alpha>0$ and $\beta<0$ we have from (1.1) and (1.3)

$$
\left\{\begin{array}{l}
\min \left\{P^{\alpha}(\Omega) T^{\beta}(\Omega):|\Omega|=m\right\}=P^{\alpha}\left(\Omega_{m}^{*}\right) T^{\beta}\left(\Omega_{m}^{*}\right) \\
\sup \left\{P^{\alpha}(\Omega) T^{\beta}(\Omega):|\Omega|=m\right\}=+\infty
\end{array}\right.
$$

and similarly, if $\alpha<0$ and $\beta>0$ we have

$$
\left\{\begin{array}{l}
\inf \left\{P^{\alpha}(\Omega) T^{\beta}(\Omega):|\Omega|=m\right\}=0 \\
\max \left\{P^{\alpha}(\Omega) T^{\beta}(\Omega):|\Omega|=m\right\}=P^{\alpha}\left(\Omega_{m}^{*}\right) T^{\beta}\left(\Omega_{m}^{*}\right) .
\end{array}\right.
$$

The cases we will investigate are the remaining ones; with no loss of generality we may assume $\alpha=1$, so that the optimization problems we consider are for the quantities

$$
P(\Omega) T^{q}(\Omega), \quad \text { with } q>0 .
$$

In order to remove the Lebesgue measure constraint $|\Omega|=m$ we consider the scaling free functionals

$$
F_{q}(\Omega)=\frac{P(\Omega) T^{q}(\Omega)}{|\Omega|^{\alpha_{q}}} \quad \text { with } \alpha_{q}=1+q+\frac{2 q-1}{d} .
$$

In the following sections we study the minimization and the maximization problems for the shape functionals $F_{q}$ on various classes of domains. More precisely we consider the cases below.

The class of all domains $\Omega$ (nonempty)

$$
\mathcal{A}_{\text {all }}=\left\{\Omega \subset \mathbb{R}^{d}: \Omega \neq \emptyset\right\}
$$

will be considered in Sect. 2; we show that for every $q>0$ both the maximization and the minimization problems for $F_{q}$ on $\mathcal{A}_{\text {all }}$ are ill posed.

The class of convex domains $\Omega$

$$
\mathcal{A}_{\text {convex }}=\left\{\Omega \subset \mathbb{R}^{d}: \Omega \neq \emptyset, \Omega \text { convex }\right\}
$$

will be considered in Sect. 3; we show that for $0<q<1 / 2$ the maximization problem for $F_{q}$ on $\mathcal{A}_{\text {convex }}$ is ill posed, whereas the minimization problem is well posed. On the contrary, when $q>1 / 2$ the minimization problem for $F_{q}$ on $\mathcal{A}_{\text {convex }}$ is ill posed, whereas the maximization problem is well posed. In the threshold case $q=1 / 2$ the precise value of the infimum of $F_{1 / 2}$ is provided; concerning the precise value of the supremum of $F_{1 / 2}$ an interesting conjecture is stated. At present, the conjecture has been shown to be true in the case $d=2$, while the question is open in higher dimensions. 
The class of thin domains $\mathcal{A}_{\text {thin }}$, suitably defined, will be considered in Sect. 4. If $h(s)$ represents the asymptotical local thickness of the thin domain as $s$ varies in a $d-1$ dimensional domain $A$, the maximization of the functional $F_{1 / 2}$ on $\mathcal{A}_{\text {thin }}$ reduces to the maximization of a functional defined on nonnegative functions $h$ defined on $A$; this allows us to prove the conjecture for any dimension $d$ on the class of thin convex domains.

\section{Optimization in the Class of All Domains}

In this section we show that the minimization and the maximization problems for the shape functionals $F_{q}$ are both ill posed, for every $q>0$.

Theorem 2.1 There exist two sequences $\left(\Omega_{1, n}\right)$ and $\left(\Omega_{2, n}\right)$ of smooth domains such that for every $q>0$ we have

$$
F_{q}\left(\Omega_{1, n}\right) \rightarrow 0 \quad \text { and } \quad F_{q}\left(\Omega_{2, n}\right) \rightarrow+\infty
$$

In particular, we have

$$
\left\{\begin{array}{l}
\inf \left\{F_{q}(\Omega): \Omega \in \mathcal{A}_{\text {all }}, \Omega \text { smooth }\right\}=0 \\
\sup \left\{F_{q}(\Omega): \Omega \in \mathcal{A}_{\text {all }}, \Omega \text { smooth }\right\}=+\infty
\end{array}\right.
$$

Proof In order to show the sup equality it is enough to take as $\Omega_{2, n}$ a perturbation of the unit ball $B_{1}$ such that

$$
B_{1 / 2} \subset \Omega_{2, n} \subset B_{2} \quad \text { and } \quad P\left(\Omega_{2, n}\right) \rightarrow+\infty .
$$

Then we have

$$
\left|\Omega_{2, n}\right| \leq\left|B_{2}\right|, \quad T\left(\Omega_{2, n}\right) \geq T\left(B_{1 / 2}\right)
$$

where we used the monotonicity of the torsional rigidity. Then

$$
F_{q}\left(\Omega_{2, n}\right) \geq \frac{P\left(\Omega_{2, n}\right) T^{q}\left(B_{1 / 2}\right)}{\left|B_{2}\right|^{\alpha_{q}}} \rightarrow+\infty .
$$

In order to prove the inf equality we take as $\Omega_{c, \varepsilon}$ the unit ball $B_{1}$ from which we remove a periodic array of holes; the centers of two adjacent holes are at distance $\varepsilon$ and the radii of the holes are

$$
r_{c, \varepsilon}= \begin{cases}e^{-1 /\left(c \varepsilon^{2}\right)} & \text { if } d=2 \\ c \varepsilon^{d /(d-2)} & \text { if } d>2\end{cases}
$$

where $c$ is a positive constant. It is easy to see that, as $\varepsilon \rightarrow 0$, we have

$$
\left|\Omega_{c, \varepsilon}\right| \rightarrow\left|B_{1}\right| \quad \text { and } \quad P\left(\Omega_{c, \varepsilon}\right) \rightarrow P\left(B_{1}\right) .
$$


Concerning the torsion $T\left(\Omega_{c, \varepsilon}\right)$, we have (see [10])

$$
T\left(\Omega_{c, \varepsilon}\right) \rightarrow \int_{B_{1}} u_{c} d x
$$

where $u_{c}$ is the nonnegative function which solves

$$
\left\{\begin{array}{l}
-\Delta u_{c}+K_{c} u_{c}=1 \text { in } B_{1} \\
u_{c} \in H_{0}^{1}\left(B_{1}\right)
\end{array}\right.
$$

being $K_{c}$ the constant

$$
K_{c}= \begin{cases}c \pi / 2 & \text { if } d=2 \\ d(d-2) 2^{-d} \omega_{d} c^{d-2} & \text { if } d>2\end{cases}
$$

Since for every $c>0$ we have that

$$
\int_{B_{1}}\left|\nabla u_{c}(x)\right|^{2}+K_{c} u_{c}^{2}(x) d x=\int_{B_{1}} u_{c} d x
$$

we get that

$$
\int_{B_{1}} u_{c} d x \leq \frac{\omega_{d}}{K_{c}}
$$

Therefore, a diagonal argument allows us to construct a sequence $\left(\Omega_{1, n}\right)$ such that

$$
\left|\Omega_{1, n}\right| \rightarrow\left|B_{1}\right|, \quad P\left(\Omega_{1, n}\right) \rightarrow P\left(B_{1}\right), \quad T\left(\Omega_{1, n}\right) \rightarrow 0,
$$

which concludes the proof.

\section{Optimization in the Class of Convex Domains}

In this section we consider only domains $\Omega$ which are convex. A first remark is in the proposition below and shows that in some cases the optimization problems for the shape functional $F_{q}$ is still ill posed.

Proposition 3.1 We have

$$
\begin{cases}\inf \left\{F_{q}(\Omega): \Omega \in \mathcal{A}_{\text {convex }}\right\}=0 & \text { for every } q>1 / 2 \\ \sup \left\{F_{q}(\Omega): \Omega \in \mathcal{A}_{\text {convex }}\right\}=+\infty & \text { for every } q<1 / 2\end{cases}
$$


Proof Let $A$ be a smooth convex $d-1$ dimensional set and for every $\varepsilon>0$ consider the domain $\Omega_{\varepsilon} \in \mathcal{A}_{\text {convex }}$ given by

$$
\left.\Omega_{\varepsilon}=A \times\right]-\varepsilon / 2, \varepsilon / 2[\text {. }
$$

We have (for the torsion asymptotics see for instance [2])

$$
\begin{aligned}
& P\left(\Omega_{\varepsilon}\right) \approx 2 \mathcal{H}^{d-1}(A), \\
& T\left(\Omega_{\varepsilon}\right) \approx \frac{\varepsilon^{3}}{12} \mathcal{H}^{d-1}(A), \\
& \left|\Omega_{\varepsilon}\right|=\varepsilon \mathcal{H}^{d-1}(A),
\end{aligned}
$$

so that

$$
F_{q}\left(\Omega_{\varepsilon}\right) \approx \frac{2}{12^{q}\left(\mathcal{H}^{d-1}(A)\right)^{(2 q-1) / d}} \varepsilon^{(2 q-1)(d-1) / d} .
$$

Letting $\varepsilon \rightarrow 0$ achieves the proof.

We show now that in some other cases the optimization problems for the shape functional $F_{q}$ is well posed. Let us begin to consider the case $q=1 / 2$.

Proposition 3.2 We have

$$
\inf \left\{F_{1 / 2}(\Omega): \Omega \in \mathcal{A}_{\text {convex }}\right\}=3^{-1 / 2}
$$

and the infimum is asymptotically reached by domains of the form

$$
\left.\Omega_{\varepsilon}=A \times\right]-\varepsilon / 2, \varepsilon / 2[
$$

as $\varepsilon \rightarrow 0$, where $A$ is any $d-1$ dimensional convex set.

Proof Thanks to a classical result by Polya ( [21], see also Theorem 5.1 of [11]) it holds

$$
T(\Omega) \geq \frac{1}{3} \frac{|\Omega|^{3}}{(P(\Omega))^{2}} .
$$

Then

$$
F_{1 / 2}(\Omega)=\frac{P(\Omega)(T(\Omega))^{1 / 2}}{|\Omega|^{3 / 2}} \geq 3^{-1 / 2}
$$

for any bounded open convex set. Taking into account (3.1), we get (3.2).

Concerning the supremum of $F_{1 / 2}(\Omega)$ in the class $\mathcal{A}_{\text {convex }}$ we can only show that it is finite. 
Proposition 3.3 For every $\Omega \in \mathcal{A}_{\text {convex }}$ we have

$$
F_{1 / 2}(\Omega) \leq \frac{2^{d} d^{3 d / 2}}{\omega_{d}} \sqrt{\frac{d}{d+2}}
$$

Proof By the John's ellipsoid Theorem [18], there exists an ellipsoid that, without loss of generality, we may assume centered at the origin,

$$
E_{a}=\left\{x \in \mathbb{R}^{d}: \sum_{i=1}^{d} \frac{x_{i}^{2}}{a_{i}^{2}}<1\right\}, \quad a=\left(a_{1}, \ldots, a_{d}\right), \text { with } a_{i}>0
$$

such that $E_{a} \subset \Omega \subset d E_{a}$. Then we have

$$
F_{1 / 2}(\Omega) \leq \frac{P\left(d E_{a}\right)\left(T\left(d E_{a}\right)\right)^{1 / 2}}{\left|E_{a}\right|^{3 / 2}} .
$$

Since the solution of (1.2) for $E_{a}$ is given by

$$
u(x)=\frac{1}{2}\left(\sum_{i=1}^{d} a_{i}^{-2}\right)^{-1}\left(1-\sum_{i=1}^{d} \frac{x_{i}^{2}}{a_{i}^{2}}\right)
$$

we obtain

$$
T\left(E_{a}\right)=\frac{\omega_{d}}{d+2}\left(\sum_{i=1}^{d} a_{i}^{-2}\right)^{-1} \prod_{i=1}^{d} a_{i}
$$

while

$$
\left|E_{a}\right|=\omega_{d} \prod_{i=1}^{d} a_{i}
$$

To estimate $P\left(E_{a}\right)$ we notice that $E_{a}$ is contained in the cuboid $\left.Q_{a}=\prod_{1}^{d}\right]-a_{i}, a_{i}[$, so that

$$
P\left(E_{a}\right) \leq P\left(Q_{a}\right)=2 \sum_{i=1}^{d} \prod_{j \neq i}\left(2 a_{j}\right)=2^{d}\left(\sum_{i=1}^{d} \frac{1}{a_{i}}\right) \prod_{i=1}^{d} a_{i} .
$$

Combining these formulas we have from (3.4)

$$
F_{1 / 2}(\Omega) \leq \frac{2^{d} d^{3 d / 2}}{\omega_{d}(d+2)^{1 / 2}}\left(\sum_{i=1}^{d} \frac{1}{a_{i}}\right)\left(\sum_{i=1}^{d} \frac{1}{a_{i}^{2}}\right)^{-1 / 2}
$$


and finally, by Jensen inequality,

$$
F_{1 / 2}(\Omega) \leq \frac{2^{d} d^{3 d / 2}}{\omega_{d}} \sqrt{\frac{d}{d+2}},
$$

as required.

On the precise value of $\sup \left\{F_{1 / 2}(\Omega): \Omega \in \mathcal{A}_{\text {convex }}\right\}$ we make the following conjecture.

Conjecture 3.4 We have

$$
\sup \left\{F_{1 / 2}(\Omega): \Omega \in \mathcal{A}_{\text {convex }}\right\}=d\left(\frac{2}{(d+1)(d+2)}\right)^{1 / 2}
$$

and it is asymptotically reached by taking for instance

$$
\Omega_{\varepsilon}=\{(s, t): s \in A, 0<t<\varepsilon(1-|s|)\}
$$

as $\varepsilon \rightarrow 0$, where $A$ is the unit ball in $\mathbb{R}^{d-1}$.

Remark 3.5 We recall that Conjecture 3.4 has been shown to be true in the case $d=2$ (see [21,23], and the more recent paper [12]). In Sect. 4 we prove the conjecture above for every $d \geq 2$ in the class of convex thin domains.

We show now that for $F_{q}$ in the class $\mathcal{A}_{\text {convex }}$ the minimization problem is well posed when $q<1 / 2$ and the maximization problem is well posed when $q>1 / 2$. From the bounds obtained in Propositions 3.2 and 3.3 we can prove the following results.

Proposition 3.6 We have

$$
\begin{cases}\inf \left\{F_{q}(\Omega): \Omega \in \mathcal{A}_{\text {convex }}\right\} \geq 3^{-1 / 2}(d(d+2))^{1 / 2-q} \omega_{d}^{(1-2 q) / d} & \text { for every } q \leq 1 / 2 \\ \sup \left\{F_{q}(\Omega): \Omega \in \mathcal{A}_{\text {convex }}\right\} \leq \frac{2^{d} d^{3 d / 2-q+1}}{(d+2)^{q} \omega_{d}^{1+(2 q-1) / d}} & \text { for every } q \geq 1 / 2 .\end{cases}
$$

Proof We have

$$
F_{q}(\Omega)=F_{1 / 2}(\Omega)\left(\frac{T(\Omega)}{|\Omega|^{(d+2) / d}}\right)^{q-1 / 2} .
$$

Hence it is enough to apply the bounds (3.2) and (3.3), together with the Saint-Venant inequality (1.3) to get that for every $\Omega \in \mathcal{A}_{\text {convex }}$

$$
\begin{aligned}
& \inf \left\{F_{q}(\Omega): \Omega \in \mathcal{A}_{\text {convex }}\right\} \geq 3^{-1 / 2}\left(\frac{T(B)}{B^{(d+2) / d}}\right)^{q-1 / 2} \quad \text { if } q \leq 1 / 2 \\
& \sup \left\{F_{q}(\Omega): \Omega \in \mathcal{A}_{\text {convex }}\right\}<\frac{2^{d} d^{3 d / 2}}{\omega_{d}} \sqrt{\frac{d}{d+2}}\left(\frac{T(B)}{B^{(d+2) / d}}\right)^{q-1 / 2} \quad \text { if } q \geq 1 / 2 .
\end{aligned}
$$


By the expression (1.4) for $T(B)$ we conclude the proof.

We now prove the existence of a convex minimizer when $q<1 / 2$ and of a convex maximizer when $q>1 / 2$.

Theorem 3.7 There exists a solution for the following optimization problems:

$$
\begin{cases}\min \left\{F_{q}(\Omega): \Omega \in \mathcal{A}_{\text {convex }}\right\} & \text { for every } q<1 / 2 \\ \max \left\{F_{q}(\Omega): \Omega \in \mathcal{A}_{\text {convex }}\right\} & \text { for every } q>1 / 2\end{cases}
$$

Proof Suppose $q<1 / 2$ and consider $\left(\Omega_{n}\right)$ a minimizing sequence for $F_{q}(\Omega)$. By the John's ellipsoid Theorem we can assume that there exists a sequence of ellipsoids $E_{a_{n}}$ such that

$$
E_{a_{n}} \subset \Omega_{n} \subset d E_{a_{n}}
$$

By rotations, translations and scaling invariance of $F_{q}$ we can assume without loss of generality that

$$
E_{a_{n}}=\left\{x \in \mathbb{R}^{d}: \sum_{i=1}^{d} \frac{x_{i}^{2}}{a_{i n}^{2}}<1\right\}, \quad a_{n}=\left(a_{1 n}, \ldots, a_{d n}\right), 0<a_{1 n} \leq \cdots \leq a_{d n}=1 .
$$

Observe that this implies that the diameter of $\Omega_{n}$ is uniformly bounded in $n$. We claim that

$$
a_{1 n} \geq c \quad \text { for every } n \in \mathbb{N}
$$

where $c$ is a positive constant. Then the proof is achieved by extracting a subsequence $\left(\Omega_{n_{k}}\right)$ which converges both in the sense of characteristic functions and in the coHausdorff metric to some open, non empty, convex, bounded set $\Omega^{-}$and by using the continuity properties of torsional rigidity, perimeter and volume (see for instance, $[7,17])$.

To prove the claim we use a strategy similar to the one already used in the proof of Proposition 3.3. Let $Q_{a_{n}}$ be the cuboid $\left.\prod_{i=1}^{d}\right]-a_{i n}, a_{i n}[$. Since

$$
d^{-1 / 2} Q_{a_{n}} \subset E_{a_{n}}
$$

we have, for $n$ large enough,

$$
F_{q}\left(B_{1}\right) \geq F_{q}\left(\Omega_{n}\right) \geq \frac{1}{d^{(d-1) / 2} d^{d \alpha_{q}}} \frac{T^{q}\left(E_{a_{n}}\right) P\left(Q_{a_{n}}\right)}{\left|E_{a_{n}}\right|^{\alpha_{q}}} .
$$

An explicit computation shows

$$
\frac{T^{q}\left(E_{a_{n}}\right) P\left(Q_{a_{n}}\right)}{\left|E_{a_{n}}\right|^{\alpha_{q}}}=\frac{2^{d} \omega_{d}^{q-\alpha_{q}}}{(d+2)^{q}}\left(\frac{\sum_{i=1}^{d} a_{i n}^{-1}}{\left(\sum_{i=1}^{d} a_{i n}^{-2}\right)^{1 / 2}}\right)\left(\frac{\left(\sum_{i=1}^{d} a_{i n}^{-2}\right)^{1 / 2}}{\left(\prod_{i=1}^{d} a_{i n}^{-1}\right)^{1 / d}}\right)^{1-2 q} .
$$


Observe that, by Cauchy-Schwarz inequality,

$$
1 \leq \frac{\sum_{i=1}^{d} a_{i n}^{-1}}{\left(\sum_{i=1}^{d} a_{i n}^{-2}\right)^{1 / 2}} \leq \sqrt{d}
$$

while for the last term it holds

$$
\frac{\left(\sum_{i=1}^{d} a_{i n}^{-2}\right)^{1 / 2}}{\left(\prod_{i=1}^{d} a_{i n}^{-1}\right)^{1 / d}}=\frac{\left(\sum_{i=1}^{d} a_{i n}^{-2}\right)^{1 / 2}}{\left(\prod_{i=1}^{d-1} a_{i n}^{-1}\right)^{1 / d}} \geq \frac{a_{1 n}^{-1}}{\left(a_{1 n}^{-1}\right)^{(d-1) / d}}=\left(\frac{1}{a_{1 n}}\right)^{1 / d}
$$

Therefore, putting together (3.5)-(3.7) and using the fact that $q<1 / 2$ we obtain that, if $n$ is large enough, the sequence $a_{1 n}$ must be greater than some positive constant $c$, which proves the claim.

The case $q>1 / 2$ can be proved in a similar way. If $\left(\Omega_{n}\right)$ is a maximizing sequence for $F_{q}(\Omega)$ and $E_{a_{n}}$ are ellipsoids such that $E_{a_{n}} \subset \Omega_{n} \subset d E_{a_{n}}$, we have

$$
F_{q}\left(B_{1}\right) \leq F_{q}\left(\Omega_{n}\right) \leq \frac{P\left(d E_{a_{n}}\right) T^{q}\left(d E_{a_{n}}\right)}{\left|E_{a_{n}}\right|^{\alpha_{q}}}=d^{d-1+q(d+2)} \frac{P\left(E_{a_{n}}\right) T^{q}\left(E_{a_{n}}\right)}{\left|E_{a_{n}}\right|^{\alpha_{q}}} .
$$

If $Q_{a_{n}}$ is the cuboid $\left.\prod_{i=1}^{d}\right]-a_{i n}, a_{i n}$ [ we have $E_{a_{n}} \subset Q_{a_{n}}$, so that

$$
P\left(E_{a_{n}}\right) \leq P\left(Q_{a_{n}}\right)=2^{d}\left(\sum_{i=1}^{d} a_{i n}^{-1}\right) \prod_{i=1}^{d} a_{i n}
$$

Hence (3.8) implies, for a suitable constant $C_{q, d}$ depending only on $q$ and on $d$,

$$
F_{q}\left(B_{1}\right) \leq C_{q, d} \frac{\sum_{i=1}^{d} a_{i n}^{-1}}{\left(\sum_{i=1}^{d} a_{i n}^{-2}\right)^{q}\left(\prod_{i=1}^{d} a_{i n}\right)^{(2 q-1) / d}} \leq d^{q} C_{q, d}\left(\frac{\left(\prod_{i=1}^{d} a_{i n}^{-1}\right)^{1 / d}}{\sum_{i=1}^{d} a_{i n}^{-1}}\right)^{2 q-1}
$$

where in the last inequality we used the Cauchy-Schwarz inequality (3.6). Finally, since $a_{\text {in }} \leq a_{d n}=1$, we obtain

$$
F_{q}\left(B_{1}\right) \leq d^{q} C_{q, d}\left(a_{i n}^{-1}\right)^{(2 q-1) / d}
$$

and, since $q>1 / 2$, the conclusion follows as in the previous case. 


\section{Optimization in the Class of Thin Domains}

In this section we consider the class of thin domains, that we define below through the families of domains

$$
\Omega_{\varepsilon}=\left\{(s, t): s \in A, \varepsilon h_{-}(s)<t<\varepsilon h_{+}(s)\right\}
$$

where $\varepsilon$ is a small positive parameter, $A$ is a (smooth) domain of $\mathbb{R}^{d-1}$, and $h_{-}, h_{+}$ are two given (smooth) functions. We denote by $h(s)$ the local thickness

$$
h(s)=h_{+}(s)-h_{-}(s)
$$

and we assume that $h(s) \geq 0$. More precisely, we call thin domain a family $\left(\Omega_{\varepsilon}\right)_{\varepsilon>0}$ as above; in other words a thin domain is characterized by the $d-1$ dimensional domain $A$ and by the local thickness function $h$.

The following asymptotics hold for the quantities we are interested to (for the torsional rigidity we refer to [5]):

$$
\begin{aligned}
& P\left(\Omega_{\varepsilon}\right) \approx 2 \mathcal{H}^{d-1}(A), \\
& T\left(\Omega_{\varepsilon}\right) \approx \frac{\varepsilon^{3}}{12} \int_{A} h^{3}(s) d s, \\
& \left|\Omega_{\varepsilon}\right|=\varepsilon \int_{A} h(s) d s
\end{aligned}
$$

which together give the asymptotic formula when $q=1 / 2$

$$
\begin{aligned}
F_{1 / 2}\left(\Omega_{\varepsilon}\right) & \approx 3^{-1 / 2} \mathcal{H}^{d-1}(A)\left[\int_{A} h^{3}(s) d s\right]^{1 / 2}\left[\int_{A} h(s) d s\right]^{-3 / 2} \\
& =3^{-1 / 2}\left[\left[f_{A} h^{3}(s) d s\right]\left[f_{A} h(s) d s\right]^{-3}\right]^{1 / 2}
\end{aligned}
$$

where we use the notation

$$
f_{A} f(s) d s=\frac{1}{\mathcal{H}^{d-1}(A)} \int_{A} f(s) d s .
$$

We then define the functional $F_{1 / 2}$ on the thin domain $\left(\Omega_{\varepsilon}\right)_{\varepsilon>0}$ associated with the $d-1$ dimensional domain $A$ and the local thickness function $h$ by

$$
F_{1 / 2}(A, h)=3^{-1 / 2}\left[\left[f_{A} h^{3}(s) d s\right]\left[f_{A} h(s) d s\right]^{-3}\right]^{1 / 2}
$$

By Hölder inequality we have

$$
F_{1 / 2}(A, h) \geq 3^{-1 / 2}
$$


and the value $3^{-1 / 2}$ is actually reached by taking the local thickness function $h$ constant, which corresponds to $\Omega_{\varepsilon}$ a thin slab.

A sharp inequality from above is also possible for $F_{1 / 2}(A, h)$, if we restrict the analysis to convex domains, that is to local thickness functions $h$ which are concave. The following result will be used, for which we refer to $[4,15]$.

Theorem 4.1 Let $1 \leq p \leq q$. Then for every convex set $A$ of $\mathbb{R}^{N}(N \geq 1)$ and every nonnegative concave function $f$ on $A$ we have

$$
\left[f_{A} f^{q} d x\right]^{1 / q} \leq C_{p, q}\left[f_{A} f^{p} d x\right]^{1 / p}
$$

where the constant $C_{p, q}$ is given by

$$
C_{p, q}=\left(\begin{array}{c}
N+p \\
N
\end{array}\right)^{1 / p}\left(\begin{array}{c}
N+q \\
N
\end{array}\right)^{-1 / q} .
$$

In addition, the inequality above becomes an equality when $A$ is a ball of radius 1 and $f(x)=1-|x|$.

We are now in a position to prove the Conjecture 3.4 for convex thin domains.

Theorem 4.2 If $\left(\Omega_{\varepsilon}\right)_{\varepsilon>0}$ is a thin convex domains given by (4.1), we have

$$
F_{1 / 2}(A, h) \leq d\left(\frac{2}{(d+1)(d+2)}\right)^{1 / 2} .
$$

In addition, the inequality above becomes an equality taking for instance as A the unit ball of $\mathbb{R}^{d-1}$ and as the local thickness $h(s)$ the function $1-|s|$.

Proof Since the local thickness function $h$ is concave, by Theorem 4.1 with $N=d-1$, $q=3, p=1$, we obtain

$$
f_{A} h^{3} d x \leq C_{1,3}^{3}\left[f_{A} h d x\right]^{3}
$$

so that

$$
F_{1 / 2}(A, h) \leq 3^{-1 / 2} C_{1,3}^{3 / 2}=d\left(\frac{2}{(d+1)(d+2)}\right)^{1 / 2}
$$

as required. Finally, an easy computation shows that in (4.3) the inequality becomes an equality if $A$ is the unit ball of $\mathbb{R}^{d-1}$ and $h(s)=1-|s|$.

Acknowledgements The work of GB is part of the project 2017TEXA3H "Gradient flows, Optimal Transport and Metric Measure Structures" funded by the Italian Ministry of Research and University. The authors are member of the Gruppo Nazionale per l'Analisi Matematica, la Probabilità e le loro Applicazioni (GNAMPA) of the Istituto Nazionale di Alta Matematica (INdAM).

Funding Information Open access funding provided by Universitá di Pisa within the CRUI-CARE Agreement. 
Open Access This article is licensed under a Creative Commons Attribution 4.0 International License, which permits use, sharing, adaptation, distribution and reproduction in any medium or format, as long as you give appropriate credit to the original author(s) and the source, provide a link to the Creative Commons licence, and indicate if changes were made. The images or other third party material in this article are included in the article's Creative Commons licence, unless indicated otherwise in a credit line to the material. If material is not included in the article's Creative Commons licence and your intended use is not permitted by statutory regulation or exceeds the permitted use, you will need to obtain permission directly from the copyright holder. To view a copy of this licence, visit http://creativecommons.org/licenses/by/4.0/.

\section{References}

1. Borell, C.: Integral inequalities for generalized concave or convex functions. J. Math. Anal. Appl. 43, 419-440 (1973)

2. Borisov, D., Freitas, P.: Asymptotics for the expected lifetime of Brownian motion on thin domains in $\mathbb{R}^{n}$. J. Theor. Probab. 26, 284-309 (2013)

3. Brasco, L.: On torsional rigidity and principal frequencies: an invitation to the Kohler-Jobin rearrangement technique. ESAIM Control Optim. Calc. Var. 20, 315-338 (2014)

4. Bucur, D., Buttazzo, G.: Variational Methods in Shape Optimization Problems, Progress in Nonlinear Differential Equations 65. Birkhäuser Verlag, Basel (2005)

5. Buttazzo, G., Guarino Lo Bianco, S., Marini, M.: Sharp estimates for the anisotropic torsional rigidity and the principal frequency. J. Math. Anal. Appl. 457, 1153-1172 (2017)

6. Cioranescu, D., Murat, F.: Un terme étrange venu d'ailleurs In: Nonlinear partial differential equations and their applications. Collège de France Seminar Vol. II, Res. Notes in Math. 60, 98-138, 389-390, Pitman, Boston (1982)

7. Crasta, G., Fragalà, I., Gazzola, F.: On a long-standing conjecture by Pólya-Szegö and related topics. Z. Angew. Math. Phys. 56, 763-782 (2005)

8. Della Pietra, F., Gavitone, N.: Sharp bounds for the first eigenvalue and the torsional rigidity related to some anisotropic operators. Math. Nachr. 287(2-3), 194-209 (2014)

9. Fragalà, I., Gazzola, F., Pierre, M.: On an isoperimetric inequality for capacity conjectured by Pólya and Szegö. J. Differ. Equ. 250, 1500-1520 (2011)

10. Fragalà, I., Gazzola, F., Lamboley, J.: Sharp bounds for the p-torsion of convex planar domains In Geometric properties for parabolic and elliptic PDE's, Springer INdAM Ser. 2, Springer Verlag, Milan, (2013), 97-115

11. Ftouhi, I., Lamboley, J.: Blaschke-Santaló diagram for volume, perimeter, and first Dirichlet eigenvaluePreprint. https://hal.archives-ouvertes.fr

12. Gardner, R.J., Zhang, G.: Affine inequalities and radial mean bodies. Am. J. Math. 120(3), 505-528 (1998)

13. Henrot, A.: Extremum Problems for Eigenvalues of Elliptic Operators. Birkhäuser Verlag, Basel (2006)

14. Henrot, A., Pierre, M.: Variation et Optimisation de Formes. Une Analyse GéométriqueMathématiques \& Applications. Springer, Berlin (2005)

15. John, F.: Extremum problems with inequalities as subsidiary conditions In: Studies and Essays Presented to R. Courant on his 60'th Birthday, Interscience Publishers, New York, 187-204 (1948)

16. Kohler-Jobin, M.T.: Une méthode de comparaison isopérimétrique de fonctionnelles de domaines de la physique mathématique. I. Première partie: une démonstration de la conjecture isopérimétrique $P \lambda^{2} \geq \pi j_{0}^{4} / 2$ de Pó1ya et SzegöZ. Angew. Math. Phys. 29, 757-766 (1978)

17. Kohler-Jobin, M.T.: Une méthode de comparaison isopérimétrique de fonctionnelles de domaines de la physique mathématique. II. Seconde partie: cas inhomogène: une inégalité isopérimétrique entre la fréquence fondamentale d'une membrane et l'énergie d'équilibre d'un problème de PoissonZ. Angew. Math. Phys. 29, 767-776 (1978)

18. Lucardesi, I., Zucco, D.: On Blaschke-Santaló diagrams for the torsional rigidity and the first Dirichlet eigenvalue. http://cvgmt.sns.it and at http://www.arxiv.org

19. Makai, E.: On the Principal Frequency of a Membrane and the Torsional Rigidity of a Beam. In Studies in Mathematical Analysis and Related Topics, pp. 227-231. Stanford University Press, Stanford (1962)

20. Pólya, G.: Two more inequalities between physical and geometrical quantities. J. Indian Math. Soc. 24, 413-419 (1960) 
21. van den Berg, M., Ferone, V., Nitsch, C., Trombetti, C.: On Pólya's inequality for torsional rigidity and first Dirichlet eigenvalue. Integr. Equ. Oper. Theory 86, 579-600 (2016)

22. van den Berg, M., Buttazzo, G., Pratelli, A.: On the relations between principal eigenvalue and torsional rigidity. Commun. Contemp. Math. http://cvgmt.sns.it and at http://www.arxiv.org

23. van den Berg, M., Buttazzo, G.: On capacity and torsional rigidity. Bull. Lond. Math. Soc. http://cvgmt. sns.it and at http://www.arxiv.org

Publisher's Note Springer Nature remains neutral with regard to jurisdictional claims in published maps and institutional affiliations. 